\title{
On the kurtosis of deep-water gravity waves
}

\author{
Francesco Fedele ${ }^{1,2} \dagger$ \\ ${ }^{1}$ School of Civil and Environmental Engineering, Georgia Institute of Technology, Atlanta, GA \\ 30322, USA \\ ${ }^{2}$ School of Electrical and Computer Engineering, Georgia Institute of Technology, Atlanta, GA \\ 30322, USA
}

(Received ?; revised ?; accepted ?. - To be entered by editorial office)

In this paper, we revisit Janssen's (2003) formulation for the dynamic excess kurtosis of weakly nonlinear gravity waves at deep water. For narrowband directional spectra, the formulation is given by a sixfold integral that depends upon the Benjamin-Feir index and the parameter $R=\sigma_{\theta}^{2} / 2 \nu^{2}$, a measure of short-crestedness for the dominant waves with $\nu$ and $\sigma_{\theta}$ denoting spectral bandwidth and angular spreading. Our refinement leads to a new analytical solution for the dynamic kurtosis of narrowband directional waves described with a Gaussian type spectrum. For multidirectional or short-crested seas initially homogenous and Gaussian, in a focusing (defocusing) regime dynamic kurtosis grows initially, attaining a positive maximum (negative minimum) at the intrinsic time scale

$$
\tau_{c}=\nu^{2} \omega_{0} t_{c}=1 / \sqrt{3 R}, \quad \text { or } \quad t_{c} / T_{0} \approx 0.13 / \nu \sigma_{\theta},
$$

where $\omega_{0}=2 \pi / T_{0}$ denotes the dominant angular frequency. Eventually the dynamic excess kurtosis tends monotonically to zero as the wave field reaches a quasi-equilibrium state characterized with nonlinearities mainly due to bound harmonics. Quasi-resonant interactions are dominant only in unidirectional or long-crested seas where the longertime dynamic kurtosis can be larger than that induced by bound harmonics, especially as the Benjamin-Feir index increases. Finally, we discuss the implication of these results on the prediction of rogue waves.

Key words: Water waves; kurtosis; nonlinear; BFI; Gaussian; focusing; rogue waves.

\section{Introduction}

Third-order quasi-resonant interactions and associated modulational instabilities cause the statistics of weakly nonlinear gravity waves to significantly differ from the Gaussian structure of linear seas (Janssen (2003); Fedele (2008); Onorato et al. (2009); Shemer \& Sergeeva (2009); Toffoli et al. (2010); Xiao et al. (2013)). One integral statistic used as a measure of the relative importance of such nonlinearities is the excess kurtosis defined by Janssen (2003) as

$$
C_{4}=\frac{\left\langle\eta^{4}\right\rangle}{3 \sigma^{4}}-1
$$

where $\eta$ is the surface displacement with respect to the mean sea level, $\sigma^{2}=\left\langle\eta^{2}\right\rangle$ is the wave variance and brackets denote statistical average. In general,

$$
C_{4}=C_{4}^{d}+C_{4}^{b},
$$

$\dagger$ Email address for correspondence: fedele@gatech.edu 
which comprises a dynamic component $C_{4}^{d}$ due to nonlinear wave-wave interactions (Janssen (2003)) and a bound contribution $C_{4}^{b}$ induced by the characteristic crest-trough asymmetry of ocean waves (see e.g. Tayfun (1980); Tayfun \& Lo (1990); Tayfun \& Fedele (2007); Fedele \& Tayfun (2009)). If third-order Stokes contributions are taken into account (Janssen \& Bidlot (2009); Janssen 2009, 2014b)

$$
C_{4}^{b}=6 \mu^{2} .
$$

For unidirectional (long-crested) seas initially homogenous and Gaussian on deep water, Mori \& Janssen (2006) have shown that the large-time behavior of the dynamic excess kurtosis is to monotonically increase towards the asymptotic value

$$
C_{4, N L S}^{d}=B F I^{2} \frac{\pi}{3 \sqrt{3}}
$$

where

$$
B F I=\frac{\mu \sqrt{2}}{\nu}
$$

is the Benjamin-Feir index,

$$
\mu=k_{0} \sigma
$$

represents an integral measure of wave steepness, $\nu$ is the spectral bandwidth and $k_{0}$ is the dominant wavenumber. The preceding approximation is valid for the dynamics of unidirectional narrowband waves described by one-dimensional (1-D) nonlinear Schrodinger (NLS) and Dysthe (1979) equations (see, for example, Shemer \& Sergeeva (2009); Shemer et al. $(2010 a \mid b))$.

Clearly, the preceding results are valid for unidirectional waves where energy is 'trapped' as in a long wave-guide. If dissipation is negligible and the wave steepness is small, quasiresonant interactions are effective in reshaping the wave spectrum, inducing nonlinear focusing and large waves in the form of breathers via modulation instability before breaking occurs (Onorato et al. (2009); Shemer \& Sergeeva (2009); Shemer et al. $(2010$ a); Chabchoub et al. (2011, 2012); Shemer \& Alperovich (2013); Shemer \& Liberzon (2014)). However, such 1-D conditions never occur in nature as they are unrealistic models of oceanic wind seas. The latter are typically multidirectional (short-crested) and energy can spread directionally. As a result, nonlinear focusing due to modulational effects is reduced (Onorato et al. (2009); Waseda et al. (2009); Toffoli et al. (2010)).

In regard to the kurtosis in short-crested seas initially homogenous and Gaussian, the focus of recent numerical studies has been on the asymptotic behavior with time (see, for example Annenkov \& Shrira (2013, 2014); Janssen \& Bidlot (2009)). Theoretical studies on the transient short-lived features of kurtosis and their relevance to the prediction of rogue waves are desirable. These provide the principal motivation for revisiting Janssen's (2003) formulation for the dynamic excess kurtosis of weakly nonlinear deep-water gravity waves.

The remainder of the paper is organized as follows. We first review Janssen's (2003) dynamic kurtosis model. Then, we present a new analytical solution of a sixfold integral that yields the growth rate of the dynamic excess kurtosis for narrowband Gaussianshaped spectra. This is followed by a detailed study of its short-time evolution and longtime asymptotic behavior and comparisons to numerical simulations and experiments. In concluding, we discuss the implications of these results on rogue wave prediction. 


\section{Dynamic excess kurtosis}

Drawing on Janssen (2003) the dynamic excess kurtosis of weakly nonlinear sea states, initially homogenous and Gaussian, is given by

$$
C_{4}^{d}=\frac{4 g}{\sigma^{2}} \operatorname{Re} \int T_{12}^{34} \delta_{12}^{34} \sqrt{\frac{\omega_{4}}{\omega_{1} \omega_{2} \omega_{3}}} G(t) E_{1} E_{2} E_{3} \mathrm{~d} \omega_{1,2,3} \mathrm{~d} \theta_{1,2,3},
$$

where the resonant function

$$
G(t)=\frac{1-\exp \left(-i \omega_{12}^{34} t\right)}{\omega_{12}^{34}}
$$

$T_{12}^{34}$ is the Zakharov kernel (Zakharov $(1968,1999)$; Krasitskii (1994)) as a function of the wavenumber vectors $\mathbf{k}_{j}=\left(k_{j} \cos \left(\theta_{j}\right), k_{j} \sin \left(\theta_{j}\right)\right)$ and $\operatorname{Re}(x)$ denotes the real part of $x$. The sixfold integral in Eq. (2.1) is defined over the manifold

$$
\mathbf{k}_{1}+\mathbf{k}_{2}-\mathbf{k}_{3}-\mathbf{k}_{4}=\mathbf{0},
$$

or equivalently $\delta_{12}^{34}=\delta\left(\mathbf{k}_{1}+\mathbf{k}_{2}-\mathbf{k}_{3}-\mathbf{k}_{4}\right)$, where $\delta(\mathbf{k})$ is the Dirac delta. The frequency mismatch is given by $\omega_{12}^{34}=\omega_{1}+\omega_{2}-\omega_{3}-\omega_{4}, E(\omega, \theta)$ is the surface spectrum and $\sigma^{2}$ is the variance of surface elevations. The deep-water angular frequency $\omega(k)=\sqrt{g k}$, the wavenumber magnitude $k=|\mathbf{k}|$ and

$$
\omega_{4}=\sqrt{g k_{4}}=\sqrt{g\left|\mathbf{k}_{1}+\mathbf{k}_{2}-\mathbf{k}_{3}\right|}
$$

follows from (2.3), with $g$ denoting gravity acceleration. Since homogenous Gaussian initial conditions with random phases and amplitudes are imposed, it follows that

$$
C_{4}^{d}(t=0)=0 .
$$

Eq. (2.1) can be simplified by resorting to a narrowband approximation (Mori \& Janssen (2006); Janssen \& Bidlot (2009)). So, we assume the spectrum $E$ to peak at $\omega=\omega_{0}$ and $\theta=\theta_{0}$, where $\omega_{0}$ and $\theta_{0}$ denote the dominant angular frequency and wave direction, respectively, and the associated wavenumber $k_{0}=\omega_{0}^{2} / g$, wave period $T_{0}=2 \pi / \omega_{0}$ and phase speed $c_{0}=\omega_{0} / k_{0}$. Next, define

$$
\omega_{j}=\omega_{0}\left(1+\nu v_{j}\right), \quad \theta_{j}=\theta_{0}+\sigma_{\theta} \phi_{j},
$$

where $\nu$ and $\sigma_{\theta}$ denote spectral and angular widths respectively. Under the narrowband condition $\nu, \sigma_{\theta} \ll 1, T_{12}^{34} \sim k_{0}^{3}$ to leading order and the frequency mismatch, correct to $O\left(\nu^{2}, \sigma_{\theta}^{2}\right)$, is given by

$$
\omega_{12}^{34} \sim \nu^{2} \omega_{0} \Delta
$$

with

$$
\Delta=\left\{\left(v_{1}-v_{3}\right)\left(v_{2}-v_{3}\right)-R\left(\phi_{1}-\phi_{3}\right)\left(\phi_{2}-\phi_{3}\right)\right\}=\Delta_{v}-R \Delta_{\phi},
$$

where $\Delta_{z}=\left(z_{1}-z_{3}\right)\left(z_{2}-z_{3}\right)$ for a generic $z=\left(z_{1}, z_{2}, z_{3}\right)$ triplet, and the parameter

$$
R=\frac{1}{2} \frac{\sigma_{\theta}^{2}}{\nu^{2}}
$$

is a measure of short-crestedness of dominant waves (Janssen \& Bidlot (2009)). Expanding Eq. (2.1) around $\nu=0$ and $\sigma_{\theta}=0$, to leading order

$$
C_{4}^{d}(\tau)=B F I^{2} J(\tau ; R),
$$

where

$$
J(\tau ; R)=2 \operatorname{Re} \int \frac{1-\exp (i \Delta \tau)}{\Delta} \widetilde{E}_{1} \widetilde{E}_{2} \widetilde{E}_{3} \mathrm{~d} v_{1,2,3} \mathrm{~d} \phi_{1,2,3}
$$


Here, $\tau=\nu^{2} \omega_{0} t$ is a dimensionless time and $\widetilde{E}_{j}\left(v_{j}, \phi_{j}\right)=E_{j} / \sigma$.

For a Gaussian-shaped spectrum, the rate of change of $C_{4}^{d}$ is explicitly given by

$$
\frac{\mathrm{d} C_{4}^{d}}{\mathrm{~d} \tau}=B F I^{2} \frac{\mathrm{d} J}{\mathrm{~d} \tau},
$$

and

$$
\frac{\mathrm{d} J}{\mathrm{~d} \tau}=\frac{\mathrm{d} J_{0}(\tau ; 1, R)}{\mathrm{d} \tau}=2 \operatorname{Im}\left(\frac{1}{\sqrt{1-2 i \tau+3 \tau^{2}} \sqrt{1+2 i R \tau+3 R^{2} \tau^{2}}}\right),
$$

where the function $J_{0}(\tau ; P, Q)$ is defined in appendix $\mathrm{A}$ and $\operatorname{Im}(x)$ denotes the imaginary part of $x$. On this basis, the factor $J$ in Eq. 2.6 follows by quadrature as

$$
J(\tau ; R)=2 \operatorname{Im} \int_{0}^{\tau} \frac{1}{\sqrt{1-2 i \alpha+3 \alpha^{2}} \sqrt{1+2 i R \alpha+3 R^{2} \alpha^{2}}} d \alpha .
$$

For small times $\tau \ll 1$,

$$
J(\tau ; R)=\int_{0}^{\tau}\left((1-R) \alpha+O\left(\alpha^{2}\right)\right) d \alpha=\frac{1}{2}(1-R) \tau^{2}
$$

and Eq. 2.6 yields

$$
C_{4}^{d} \sim B F I^{2}(1-R) \tau^{2}, \quad \tau \ll 1,
$$

in agreement with Janssen \& Bidlot (2009).

Note that the dynamic excess kurtosis in Eq. 2.6 is consistent with the evolution of weakly nonlinear narrowband wavetrains of the two-dimensional (2-D) NLS equation.

\section{Intrinsic nonlinear time scale}

The growth rate 2.8 of the dynamic $C_{4}^{d}$ vanishes at the dimensionless time

$$
\tau_{c}=\frac{1}{\sqrt{3 R}}
$$

or in physical units

$$
\frac{t_{c}}{T_{0}}=\frac{1}{2 \pi} \sqrt{\frac{2}{3}} \frac{1}{\sigma_{\theta} \nu} \sim \frac{0.13}{\sigma_{\theta} \nu},
$$

where $T_{0}=2 \pi / \omega_{0}$ is the dominant wave period. Further, the second derivative of $C_{4}^{d}$ at $\tau_{c}$ is given by

$$
\left.\frac{\mathrm{d}^{2} C_{4}^{d}}{\mathrm{~d} \tau^{2}}\right|_{\tau=\tau_{c}}=-6 \sqrt{3} R(1-R) .
$$

Thus, $C_{4}^{d}$ attains a positive maximum (negative minimum) at $\tau=\tau_{c}$ for $0<R<1$ $(R>1)$. It is straightforward to show that for multidirectional or short-crested seas $(R>0)$

$$
\lim _{\tau \rightarrow \infty} C_{4}^{d}=0 .
$$

Indeed, it is sufficient to study the rate of change of $C_{4}^{d}$ for large times $\tau \gg 1$. To do so, consider the change of variable $\tau=1 / r$ and expanding (2.8) around $r=0$ yields

$$
\frac{\mathrm{d} C_{4}^{d}}{\mathrm{~d} \tau} \sim \frac{(-1+R) r^{3}}{9 R^{2}}=\frac{(-1+R)}{9 R^{2} \tau^{3}} .
$$

Note that the real part of Eq. (2.8), which has no physical meaning, decays as $\tau^{-2}$. For $0<R<1, C_{4}^{d}$ first attains a positive peak at $\tau=\tau_{c}$ and then decays monotonically 


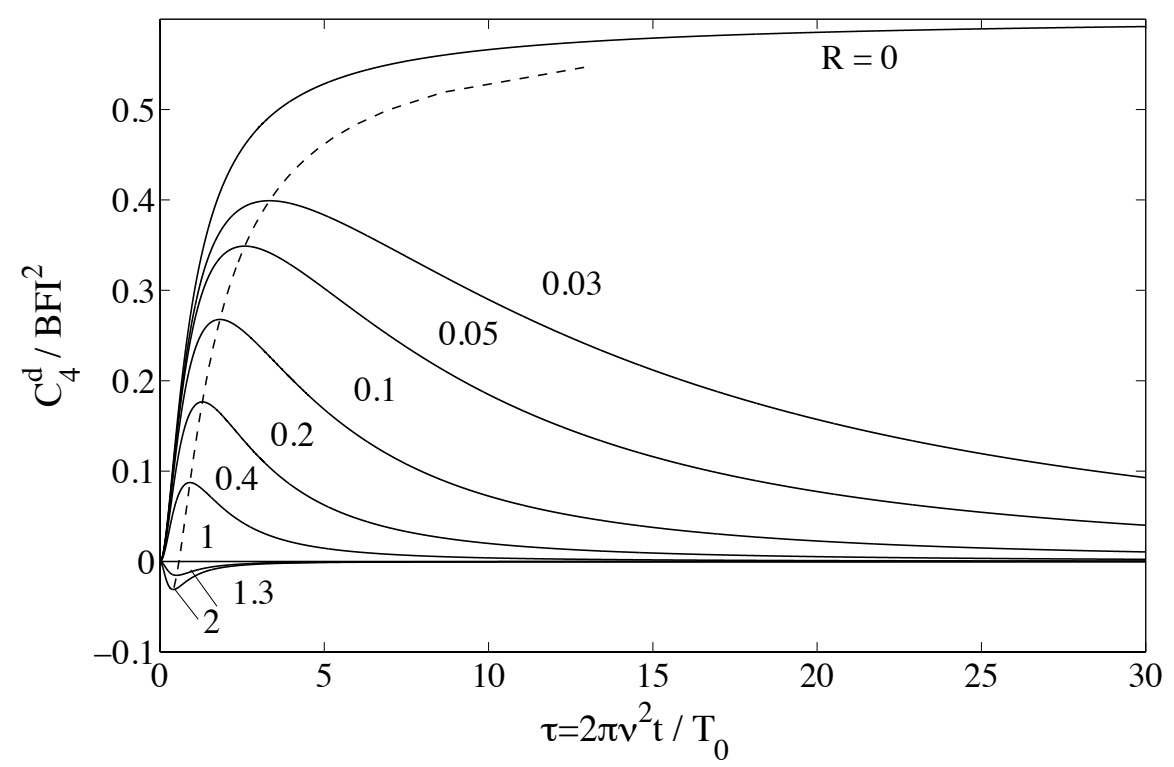

$[\mathrm{H}]$

Figure 3.1. Dynamic excess kurtosis: (solid lines) $C_{4}^{d} / B F I^{2}$ as a function of dimensionless time $\tau=\nu^{2} \omega_{0} t=2 \pi \nu^{2} t / T_{0}$ for different values of $R$; (dashed line) locus of transient peaks $\left(T_{0}\right.$ denotes the dominant wave period and $\nu$ is the spectral bandwidth).

to zero since $\mathrm{d} C_{4}^{d} / \mathrm{d} \tau<0$ for large $\tau$. This is clearly seen in Figure (3.1), showing the evolution of $C_{4}^{d}$ for different values of $R$. For $R>1, C_{4}^{d}$ initially decreases reaching a negative peak at $\tau=\tau_{c}$ and then tends monotonically to zero, because $\mathrm{d} C_{4}^{d} / \mathrm{d} \tau>0$ for large $\tau$ as shown in Figure (3.1). At the critical value $R=1$, the excess kurtosis is null at any time, as can easily be verified from Eq. 2.8.

In summary, depending on the value of $R$ there will be nonlinear focussing $\left(C_{4}^{d}>0\right)$ or nonlinear defocussing $\left(C_{4}^{d}<0\right)$ in agreement with Janssen \& Bidlot (2009). Note that for unidirectional or long-crested seas $(R=0)$ the rate of change $\mathrm{d} C_{4}^{d} / \mathrm{d} \tau>0$ for any time $\tau$. In this case, the dynamic excess kurtosis monotonically increases with time to the asymptotic value of Eq. 1.4) (Mori \& Janssen (2006); Shemer \& Sergeeva (2009); Shemer et al. $(2010 a$ b $)$; Fedele et al. $(2010))$.

\section{Dynamic excess kurtosis maximum}

From Eqs. 2.6 and 3.1, the peak value of $C_{4}^{d}$ at $\tau=\tau_{c}$ is given by

$$
C_{4}^{d}(R)=B F I^{2} J_{p}(R)
$$

where

$$
J_{p}(R)=J\left(\frac{1}{\sqrt{3 R}} ; R\right)=\operatorname{Im} \int_{0}^{\frac{1}{\sqrt{3 R}}} \frac{2}{\sqrt{1-2 i \alpha+3 \alpha^{2}} \sqrt{1+2 i R \alpha+3 R^{2} \alpha^{2}}} d \alpha .
$$

The following relation holds

$$
J_{p}\left(\frac{1}{R}\right)=-R J_{p}(R)
$$


in agreement with Janssen \& Bidlot (2009). This relation allows us to compute the minimum kurtosis for $R>1$ from the maximum value for $R<1$. Indeed,

$$
C_{4, \min }^{d}\left(\frac{1}{R}\right)=-R C_{4, \max }^{d}(R), \quad 0 \leqslant R \leqslant 1 .
$$

where $C_{4, \max }^{d}=B F I^{2} J_{p}(R)$. Clearly, this vanishes at $R=1$ signaling the change from a nonlinear focusing to defocusing regime where the dynamic kurtosis is negative.

Drawing on Janssen \& Bidlot (2009), the limit

$$
J_{p}(R) \sim-\frac{\pi}{3 \sqrt{3} R}, \quad R \gg 1
$$

and that for small-times in Eq. 2.11) suggest the least-squares fit for the maximum

$$
\frac{C_{4, \max }^{d}(R)}{B F I^{2}}=J_{\text {peak }}(R) \approx \frac{b}{(2 \pi)^{2}} \frac{1-R}{R+b R_{0}}, \quad 0 \leqslant R \leqslant 1,
$$

where $R_{0}=\frac{3 \sqrt{3}}{\pi}$ and $b=2.48$. In the left panel of Figure 4.1, the preceding approximation is compared against the theoretical $C_{4, \max }^{d}$ solving Eq. 4.1 by numerical integration. Evidently, the latter is slightly larger than the maximum excess kurtosis derived by Janssen \& Bidlot (2009), who have also used (4.4) but with $b=1$. Their maximum follows by first taking the limit of the resonant function $G(t)$ in Eq. 2.2 at $t=\infty$ and then solving the sixfold integral in Eq. 2.1). Clearly, for $R>0$ the dynamic excess kurtosis should vanish at large times as discussed above. Janssen (personal communication, 2014) confirmed that Eq. 4.1 holds and provided an alternative proof that $C_{4}^{d}$ tends to zero as $t \rightarrow \infty$ using complex analysis and numerical integration.

Further, from 3.1

$$
\frac{C_{4, \max }^{d}\left(\tau_{c}\right)}{B F I^{2}} \approx \frac{b}{(2 \pi)^{2}} \frac{-1+3 \tau_{c}^{2}}{1+3 b R_{0} \tau_{c}^{2}}, \quad 0 \leqslant \tau_{c} \leqslant \frac{1}{\sqrt{3}} .
$$

Clearly, the transient maximum kurtosis becomes larger for longer time scales $\tau_{c}$, as illustrated in the right panel of Figure 4.1. Note that the dynamic excess kurtosis is negative for $\tau_{c}>1 / \sqrt{3}$ as the wave regime is of defocusing type $(R>1)$ and the minimum value $C_{4, \min }^{d}$ can be computed from Eq. 4.2.

\section{Comparisons to simulations and experiments}

We now compare the theoretical narrowband (NB) predictions for the total kurtosis $C_{4}$ [see Eqs. [1.2), (2.6) and (1.3)] to experimental results $($ Onorato et al. $(2009))$ and the comprehensive numerical simulations of JONSWAP directional wave fields carried out by Xiao et al. (2013) and Toffoli et al. (2010). They considered the broad-band modified nonlinear Schrodinger equations (BMNLS) (Dysthe $(1979))$ and a high-order spectral (HOS) solver (Dommermuth \& Yue (1987)). In particular, we consider the comprehensive numerical results reported in Figs. 10a,b in Xiao et al. (2013) for the two cases of narrow and broad directional spreading, i.e. $\sigma_{\theta}=0.04$ and 0.07 respectively. The simulated sea states have standard deviation $\sigma=0.02 \mathrm{~m}$, dominant wave period $T_{0}=1 \mathrm{~s}$, significant wave height $H_{s}=4 \sigma=0.08 \mathrm{~m}, B F I=0.78$, wave steepness $\mu=0.08$ and spectral bandwidth $\nu=0.15$ (see appendix B for the estimation of wave parameters). As shown in Figure 5.1, the numerical studies by Xiao et al. (2013) indicate an initial overshoot of the kurtosis followed by a decay towards quasi-Gaussian conditions.

In particular, the left panel of Figure 5.1 shows that for a narrow directional spreading 
$[\mathrm{H}]$
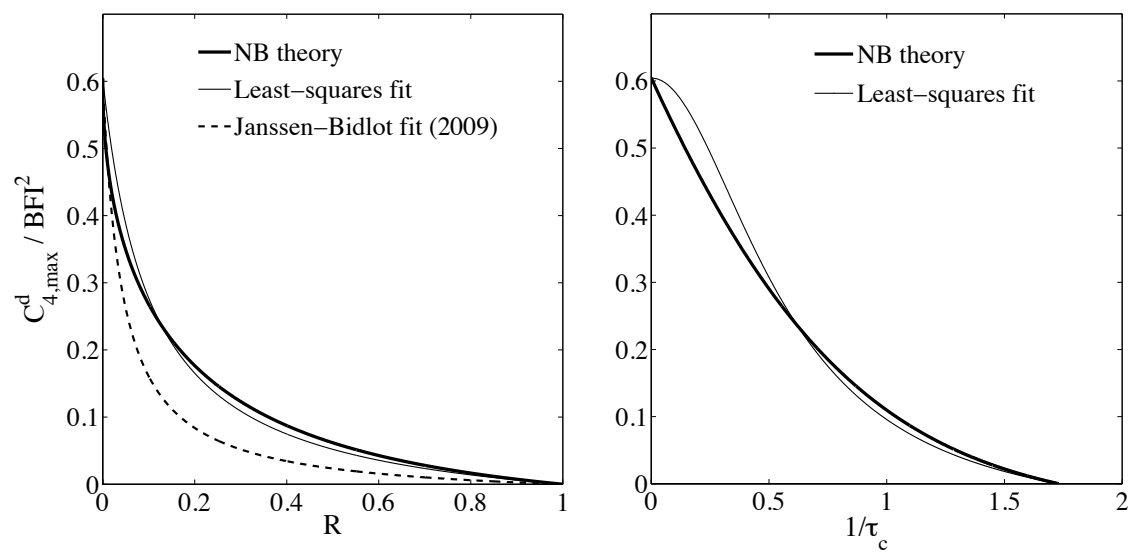

Figure 4.1. Maximum dynamic excess kurtosis $C_{4, \max }^{d}$ as a function of (left) $R$ and (right) $1 / \tau_{c}$ : (bold line) present theoretical prediction, (thin line) least-squares fit from Eq. $4.4(b=2.48)$ and (dash line) Janssen-Bidlot (2009) fit $(b=1)$.

$\left(\sigma_{\theta} \sim 0.04\right)$ the present theoretical NB model (thick line) explains the peak kurtosis and the initial transient behavior of BMNLS simulations (thin dashed line) as NB is consistent with the dynamics of the 2-D NLS equation. BMNLS and NB yield faster initial growth and overestimate both HOS (thin solid line) and experiments (triangle symbols). However, soon after the transient stage, the spectrum has already broadened in frequency and spread angularly, approaching a quasi-equilibrium state. At this stage, the NB approximation provides just a qualitative trend of the large-time behavior since it does not account for spectral changes. In particular, NB shows a slower decaying trend to zero than BMNLS. This indicates that numerical models capture the directional energy spreading and quasi-resonant interactions attenuate much faster than NB after the transient peak.

For a broad directional spreading $\left(\sigma_{\theta} \sim 0.07\right)$ the right panel of Figure 5.1 shows that NB overestimates the maximum kurtosis and qualitatively explains the initial transient overshoot of BMNLS simulations, which are now beyond their range of validity as the spectrum is already too broad initially. Instead, HOS simulations are in agreement with experiments and yield a smaller value of the maximum kurtosis and a slower transient than BMNLS.

In both the abovementioned cases, the NB model qualitatively describes the initial transient and kurtosis peak. For time scales $t \gg t_{c}$, NB indicates the correct asymptotic behavior of the total kurtosis of surface elevations as dominated by nonlinear bound harmonics (see also Annenkov \& Shrira (2013, 2014)).

\section{Concluding remarks}

Our refinement of Janssen's (2003) formulation leads to a new analytical solution for the dynamic excess kurtosis of weakly nonlinear deep-water gravity waves and associated growth rate. It assumes that waves are approximately narrowband and characterized with a Gaussian type spectrum that does not change over time.

For short-crested seas initially homogenous and Gaussian, in the focusing regime $(0<$ $R<1$ ) the dynamic excess kurtosis initially grows attaining a maximum at the intrinsic 
$[\mathrm{H}]$
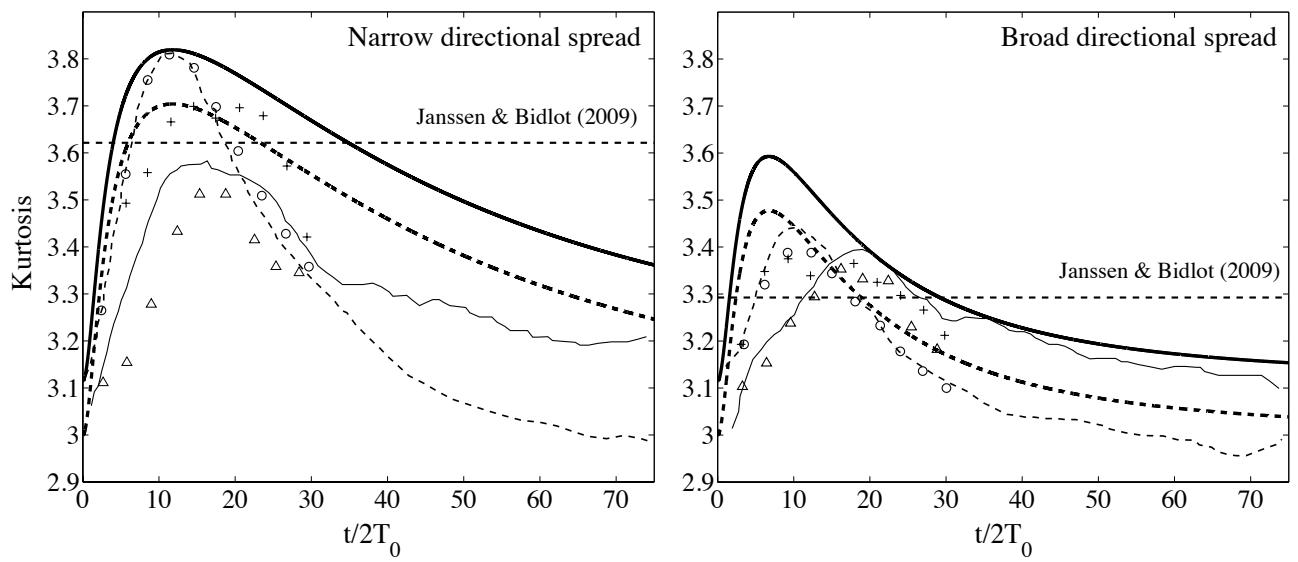

Figure 5.1. Kurtosis $\mu_{4}=\left\langle\eta^{4}\right\rangle /\left\langle\eta^{2}\right\rangle^{2}=3\left(C_{4}+1\right)$ as a function of time $t / 2 T_{0}$ for JONSWAP directional wave fields initially homogenous and Gaussian $(B F I=0.78, \mu=0.08, \nu=0.15)$ : theoretical narrowband predictions compared against simulations and experiments $(\triangle)$ from Onorato et al. (2009) (data digitized from Fig. 10a,b in Xiao et al. (2013)). (Left) narrow directional spreading with $\sigma_{\theta}=0.04, R=0.03$ (see Eq. 2.5) and (Right) broad directional spreading with $\sigma_{\theta}=0.07, R=0.1$. Narrowband theory: dynamic kurtosis $\mu_{4}^{d}=3\left(C_{4}^{d}+1\right)$ from Eq. (2.6) (thick dashed line) and total kurtosis $\mu_{4}=\mu_{4}^{d}+\mu_{4}^{b}$ (thick solid line), with $\mu_{4}^{b}=3\left(C_{4}^{b}+1\right)$ from Eq. (1.3). Dashed horizontal lines denote Janssen \& Bidlot (2009) dynamic kurtosis maximum from Eq. 4.4 with $b=1$. Simulation results from Xiao et al. (2013): HOS (thin solid line), BMNLS (thin dashed line). The numerical results from Toffoli et al. $[(2010)$ are also shown: $\operatorname{BMNLS}(\bigcirc)$ and $\operatorname{HOS}(+)$.

time scale

$$
\tau_{c}=\nu^{2} \omega_{0} t_{c}=\frac{1}{\sqrt{3 R}}, \quad \text { or } \quad \frac{t_{c}}{T_{0}} \sim \frac{0.13}{\nu \sigma_{\theta}} .
$$

Eventually it tends monotonically to zero as energy spreads directionally. In the defocusing regime $(R>1)$ the dynamic excess kurtosis is always negative attaining a minimum at $t_{c}$ and then it tends to zero in the long time. Thus, the present theoretical narrowband predictions indicate a decaying trend for the dynamic excess kurtosis over large times. This implies that for time scales $t \gg t_{c}$ the asymptotic behavior of the total kurtosis of surface elevations is dominated by nonlinear bound harmonics in qualitatively accord with numerical simulations (Annenkov \& Shrira (2009); Toffoli et al. (2010); Xiao et al. (2013); Annenkov \& Shrira (2014)) and experiments (Onorato et al. (2009); Waseda et al. $(2009))$. For time scales of the order of or less than $t_{c}$ the dynamic component can dominate and the wave field may experience rogue wave behavior induced by quasi-resonant interactions (Janssen $(2003))$.

Current statistical approaches for freak wave warning systems and predictions rely on the Gram-Charlier type probability distribution for crest-to-trough wave heights $z=H / \sigma$ ( see, e.g. Janssen (2003); Mori \& Janssen (2006); Tayfun \& Fedele (2007))

$$
\operatorname{Pr}\{H / \sigma>z\}=\left[1+\frac{C_{4}}{384} z^{2}\left(z^{2}-16\right)\right] \exp \left(-\frac{z^{2}}{8}\right)
$$

and Janssen's (2003) theory for the kurtosis $C_{4}$, a key result with significant implications to the understanding of the role of nonlinear wave interactions (Janssen (2003, 2009)). The present study suggests that it is important to reconsider it carefully. Indeed, the large excess kurtosis transient observed during the initial stage is a result of the unrealistic 
assumption that the initial wave field is homogeneous Gaussian. A random wave field forgets its initial conditions and adjusts to a non-Gaussian state dominated by bound nonlinearities on time scales $t \gg t_{c}$ in agreement with experiments (Onorato et al. (2009); Waseda et al. (2009)) and simulations (Annenkov \& Shrira (2013, 2014)). In this regime, statistical prediction of extreme waves can be based on the Tayfun (1980) model (Tayfun \& Fedele (2007); Fedele (2008); Fedele \& Tayfun (2009); Fedele (2015)).

The NB approximation for kurtosis is only consistent with the dynamics of the 2D NLS equation. It just qualitatively captures the transient behavior of the kurtosis observed in wave tank experiments due to a cold start, i.e. initially when the wave field is homogenous and Gaussian (Onorato et al. (2009)). Instead, it tends to agree with numerical simulations of the BMNLS equations for narrowband spectra (Toffoli et al. (2010); Xiao et al. (2013)). And, for time scales $t>t_{c}$ NB theory indicates the correct asymptotic behavior of the total kurtosis of surface elevations as dominated by bound harmonic contributions.

Further, NB predictions tend to overestimate the observed kurtosis maximum in wave tank experiments (see Fig. (5.1)) suggesting that higher order nonlinearities and broader spectral bandwidth effects should be accounted for in the theoretical analysis. Indeed, for the compact form of the 1-D Zakharov equation (cDZ, Dyachenko \& Zakharov (2011)), Fedele (2014) showed that, correct to $O\left(\nu^{2}\right)$ in spectral bandwidth, the associated maximum dynamic kurtosis

$$
C_{4, c D Z}^{d}=C_{4, N L S}^{d}\left(1-\frac{4 \sqrt{3}+\pi}{8 \pi} \nu^{2}\right) \approx C_{4, N L S}^{d}\left(1-0.40 \nu^{2}\right)
$$

is smaller than the NLS counterpart $C_{4, N L S}^{d}$ in Eq. 1.4, especially as the spectrum widens. The present study can be extended to derive an analytical solution of the kurtosis evolution from a cold start in accord with the 2-D Zakharov equation (Dyachenko \& Zakharov (2011); Gramstad (2014)), but this is beyond the scope of this work.

Thus, the present theoretical results for the third-order nonlinear statistics of wave fields characterized by a narrow spectrum of Gaussian shape are not relevant for predictions of extreme waves in realistic oceanic seas. It appears that such results may just provide a qualitative trend of the short-time kurtosis behavior induced by a cold start in the context of experiments in wave tanks.

\section{Acknowledgments}

FF is grateful to Peter A. E. M. Janssen for suggesting the topic of this work and for discussions on nonlinear water waves. FF also thanks Michael Banner, Victor Shrira and M. Aziz Tayfun for discussions on nonlinear wave statistics and random wave fields.

\section{Appendix A}

Consider the generic sixfold integral

$$
J_{0}(\tau ; P, Q)=2 \operatorname{Re} \int \frac{1-\exp (i \Delta \tau)}{\Delta} \widetilde{E}_{1} \widetilde{E}_{2} \widetilde{E}_{3} \mathrm{~d} v_{1,2,3} \mathrm{~d} \phi_{1,2,3}
$$

where $P$ and $Q$ are complex coefficients,

$$
\Delta=P \Delta_{v}-Q \Delta_{\phi},
$$


and

$$
\widetilde{E}_{j}\left(v_{j}, \phi_{j}\right)=\frac{\exp \left(-\frac{v_{j}^{2}+\phi_{j}^{2}}{2}\right)}{2 \pi} .
$$

Then, the integral (8.1) can be written as

$J_{0}(\tau ; P, Q)=\operatorname{Re} \int \frac{1-\exp \left(i P \Delta_{v} \tau-i Q \Delta_{\phi} \tau\right)}{P \Delta_{v}-i Q \Delta_{\phi}} \frac{\exp \left(-\frac{v_{1}^{2}+v_{2}^{2}+v_{3}^{2}}{2}\right)}{(2 \pi)^{3 / 2}} \frac{\exp \left(-\frac{\phi_{1}^{2}+\phi_{2}^{2}+\phi_{3}^{2}}{2}\right)}{(2 \pi)^{3 / 2}} d v_{1,2,3} d \phi_{1,2,3}$

Clearly, $v_{j}$ and $\phi_{j}$ are coupled via the denominator $P \Delta_{v}-i Q \Delta_{\phi}$. However, they become uncoupled if we take the time derivative

$$
\frac{d J_{0}}{d \tau}=\operatorname{Im} \int \exp \left(i P \Delta_{v} \tau-i Q \Delta \tau\right) \frac{\exp \left(-\frac{v_{1}^{2}+v_{2}^{2}+v_{3}^{2}}{2}\right)}{(2 \pi)^{3 / 2}} \frac{\exp \left(-\frac{\phi_{1}^{2}+\phi_{2}^{2}+\phi_{3}^{2}}{2}\right)}{(2 \pi)^{3 / 2}} d v_{1,2,3} d \phi_{1,2,3}
$$

Indeed,

$$
\frac{d J_{0}}{d \tau}=2 \operatorname{Im}\left[I_{0}(\tau ; P) I_{0}(\tau ;-Q)\right]
$$

where

$$
I_{0}(\tau ; P)=\int \exp \left(i P \Delta_{z} \tau\right) \frac{\exp \left(-\frac{z_{1}^{2}+z_{2}^{2}+z_{3}^{2}}{2}\right)}{(2 \pi)^{3 / 2}} d z_{1,2,3}
$$

Drawing on Fedele et al. (2010) Gaussian integration yields

$$
I_{0}(\tau ; P)=\frac{1}{\sqrt{1-2 i P \tau+3 P^{2} \tau^{2}}}
$$

and from 8.3

$$
\frac{\mathrm{d} J_{0}(\tau ; P, Q)}{\mathrm{d} \tau}=2 \operatorname{Im}\left(\frac{1}{\sqrt{1-2 i P \tau+3 P^{2} \tau^{2}} \sqrt{1+2 i Q \tau+3 Q^{2} \tau^{2}}}\right) .
$$

\section{Appendix B}

Recently, Xiao et al. (2013) and Toffoli et al. (2010) have compared BMNLS and HOS simulations of JONSWAP directional wave fields to the experimental results in Onorato et al. (2009). Their Benjamin-Feir index is a factor $\sqrt{2}$ larger than the one used in this work [see Eq. [1.5]], that is

$$
B F I^{\prime}=\frac{2 k_{0} \sigma}{\nu}=\frac{2 \mu}{\nu}=\sqrt{2} B F I .
$$

Further, their wave steepness $\mu^{\prime}=2 \mu$ where $\mu=k_{0} \sigma$ is used in this work (see also Table 1 in Toffoli et al. (2010)). In the numerical results reported in Fig. 10a,b of Xiao et al. (2013), $B F I^{\prime}=1.1$ and $\mu^{\prime}=0.16$. Thus, $B F I=0.78, \mu=0.08$ and the spectral bandwidth follows as $\nu=\sqrt{2} \mu / B F I=0.15$.

The directional distribution $D(\theta)$ adopted by Xiao et al. (2013) is given by

$$
D(\theta)=\frac{2}{\Theta} \cos ^{2}\left(\frac{\pi \theta}{\Theta}\right), \quad|\theta| \leqslant \frac{\Theta}{2},
$$


and the associated directional spreading follows as

$$
\sigma_{\theta}=\sqrt{\frac{\int_{-\frac{\Theta}{2}}^{\frac{\Theta}{2}} D(\theta) \theta^{2} d \theta}{\int_{-\frac{\Theta}{2}}^{\frac{\Theta}{2}} D(\theta) d \theta}}=\Theta \sqrt{\frac{\pi^{2}-6}{12 \pi^{2}}} .
$$

The numerical results shown in Fig. 10a of Xiao et al. $(2013)$ are for $\Theta=12 \frac{\pi}{180} \mathrm{rad}$ (narrow directional spreading); using Eq. (9.1) yields $\sigma_{\theta}=0.04$ and $R=0.03$ from Eq. 2.5. For the case of broad directional spreading shown in their Fig. $10 \mathrm{~b} \Theta=21 \frac{\pi}{180} \mathrm{rad}$ and $\sigma_{\theta}=0.07, R=0.1$.

\section{REFERENCES}

Annenkov, S. Y. \& Shrira, V. I. 2009 Evolution of kurtosis for wind waves. Geophysical Research Letters 36 (13), 1944-8007.

Annenkov, S. Y. \& Shrira, V. I. 2013 Large-time evolution of statistical moments of windwave fields. Journal of Fluid Mechanics 726, 517-546.

Annenkov, S. Y. \& Shrira, V. I. 2014 Evaluation of skewness and kurtosis of wind waves parameterized by jonswap spectra. Journal of Physical Oceanography 44 (6), 1582-1594.

Chabchoub, A., Hoffmann, N., Onorato, M. \& Akhmediev, N. 2012 Super rogue waves: Observation of a higher-order breather in water waves. Phys. Rev. X 2, 011015.

Chabchoub, A., Hoffmann, N. P. \& Akhmediev, N. 2011 Rogue wave observation in a water wave tank. Phys. Rev. Lett. 106, 204502.

Dommermuth, D. G. \& Yue, D. K. P. 1987 A high-order spectral method for the study of nonlinear gravity waves. Journal of Fluid Mechanics 184, 267-288.

Dyachenko, A. I. \& Zakharov, V. E. 2011 Compact Equation for Gravity Waves on Deep Water. JETP Lett. 93 (12), 701-705.

Dysthe, K. B. 1979 Note on a modification to the nonlinear Schrödinger equation for application to deep water. Proc. R. Soc. Lond. A 369, 105-114.

Fedele, F. 2008 Rogue waves in oceanic turbulence. Physica D 237, 2127-2131.

Fedele, F. 2014 On certain properties of the compact zakharov equation. Journal of Fluid Mechanics 748, 692-711.

Fedele, F. 2015 On oceanc rogue waves, http://arxiv.org/abs/1501.03370.

Fedele, F., Cherneva, Z., Tayfun, M. A. \& Soares, C. Guedes 2010 Nonlinear schrodinger invariants and wave statistics. Physics of Fluids 22 (3), 036601.

Fedele, F. \& TAyfun, M. A. 2009 On nonlinear wave groups and crest statistics. J. Fluid Mech 620, 221-239.

Gramstad, ODIN 2014 The zakharov equation with separate mean flow and mean surface. Journal of Fluid Mechanics 740, 254-277.

Janssen, P.A.E.M. 2009 On some consequences of the canonical transformation in the Hamiltonian theory of water waves. J. Fluid Mech 637, 1-44.

Janssen, P. A. E. M. 2003 Nonlinear four-wave interactions and freak waves. Journal of Physical Oceanography 33 (4), 863-884.

Janssen, Peter A. E. M. $2014 a$ Notes on kurtosis evolution for 2 d wave propagation. Memorandum Research Department 60.9/PJ/0387. ECMWF.

Janssen, Peter A. E. M. $2014 b$ On a random time series analysis valid for arbitrary spectral shape. Journal of Fluid Mechanics 759, 236-256.

Janssen, Peter A. E. M. \& Bidlot, J. R. 2009 On the extension of the freak wave warning system and its verification. Tech. Memo 588. ECMWF.

KrasitskiI, V. P. 1994 On reduced equations in the Hamiltonian theory of weakly nonlinear surface waves. J. Fluid Mech 272, 1-20.

Mori, N. \& Janssen, P. A. E. M. 2006 On kurtosis and occurrence probability of freak waves. Journal of Physical Oceanography 36 (7), 1471-1483.

Onorato, M., Cavaleri, L., Fouques, S., Gramstad, O., Janssen, P.A.E.M., Monbaliu, J., Osborne, A. R., Pakozdi, C., Serio, M., Stansberg, C. T., Toffoli, A. \& Trulsen, K. 2009 Statistical properties of mechanically generated surface grav- 
ity waves: a laboratory experiment in a three-dimensional wave basin. Journal of Fluid Mechanics 627, 235-257.

Shemer, L. \& Alperovich, S.H. 2013 Peregrine breather revisited. Physics of Fluids 25, 051701.

Shemer, L. \& Liberzon, D. 2014 Lagrangian kinematics of steep waves up to the inception of a spilling breaker. Physics of Fluids 26 (1), 016601.

Shemer, L. \& Sergeeva, A. 2009 An experimental study of spatial evolution of statistical parameters in a unidirectional narrow-banded random wavefield. Journal of Geophysical Research: Oceans 114 (C1), 2156-2202.

Shemer, Lev, Sergeeva, Anna \& Liberzon, Dan $2010 a$ Effect of the initial spectrum on the spatial evolution of statistics of unidirectional nonlinear random waves. Journal of Geophysical Research: Oceans 115 (C12), n/a-n/a.

Shemer, Lev, Sergeeva, Anna \& Slunyaev, Alexey $2010 b$ Applicability of envelope model equations for simulation of narrow-spectrum unidirectional random wave field evolution: Experimental validation. Physics of Fluids (1994-present) 22 (1), -.

TAyfun, M. AzIZ 1980 Narrow-band nonlinear sea waves. Journal of Geophysical Research: Oceans 85 (C3), 1548-1552.

Tayfun, M. A. \& Fedele, F. 2007 Wave-height distributions and nonlinear effects. Ocean Engineering 34 (11-12), 1631 - 1649.

TAYfun, M. A. \& Lo, J. 1990 Nonlinear effects on wave envelope and phase. J. Waterway, Port, Coastal and Ocean Eng. 116, 79-100.

Toffoli, A., Gramstad, O., Trulsen, K., Monbaliu, J., Bitner-Gregersen, E. \& Onorato, M. 2010 Evolution of weakly nonlinear random directional waves: laboratory experiments and numerical simulations. Journal of Fluid Mechanics 664, 313-336.

Waseda, T., Kinoshita, T. \& Tamura, H. 2009 Evolution of a random directional wave and freak wave occurrence. Journal of Physical Oceanography 39 (3), 621-639.

Xiao, W., Liu, Y., Wu, G. \& Yue, D. K. P. 2013 Rogue wave occurrence and dynamics by direct simulations of nonlinear wave-field evolution. Journal of Fluid Mechanics 720, 357-392.

Zakharov, V. E. 1968 Stability of periodic waves of finite amplitude on the surface of a deep fluid. J. Appl. Mech. Tech. Phys. 9, 190-194.

ZAKHAROV, V. E. 1999 Statistical theory of gravity and capillary waves on the surface of a finite-depth fluid. Eur. J. Mech. B/Fluids 18 (3), 327-344. 